\title{
El método del caso: una herramienta de gran utilidad
}

\author{
Carlos Cienfuegos Alvarado ${ }^{1}$ \\ Delia G. Gómez Morales² \\ María Antonieta Rebeil Corella ${ }^{3}$ \\ https://doi.org/10.36105/stx.2018n1.05
}

\section{Resumen}

El método de casos es una técnica de aprendizaje que tuvo su origen en la Universidad de Harvard, específicamente en la Escuela de Derecho, a principios del siglo pasado. En el transcurso de los años se extendió hacia Europa, Asia y América Latina, donde ha sido y es utilizado por diversas escuelas y facultades en centros de investigación y universidades de prestigio. Se trata de una metodología de aprendizaje activo, en donde el alumno se enfrenta a un problema real y específico y comprende, conoce y analiza el contexto en el que se presenta. Al utilizar esta metodología se ve beneficiado tanto el catedrático como el alumno,

1 Director de la Facultad de Comunicación de la Universidad Anáhuac México. Maestro en Dirección de Empresas del Instituto Panamericano de Alta Dirección de Empresas (IPADE) y candidato a doctor en Administración Pública por la Universidad Anáhuac México y la Universidad Oarus XIII, Sorbona, París Cité. Cursó el programa The Art Craft of Discussion Leadersbip en Harvard Business School y es facilitador certificado en la Metodología LEGo Serious Play. carlos.cienfuegos@anahuac.mx

2 Profesora de posgrado en la Facultad de Comunicación y de Responsabilidad Social de la Universidad Anáhuac México e investigadora asociada en el Centro de Investigación para la Comunicación Aplicada (CICA). Doctora en Comercio Internacional por la Universidad de Florida. Maestra en Relaciones México Estados Unidos por la unam. Co-coordinadora de cuatros textos y autora del libro Cazadores de series televisivas: análisis e impacto.deliagomez@prodigy.net.mx

3 Directora del Centro de Investigación para la Comunicación Aplicada (CICA), Coordinadora Académica del Doctorado en Comunicación Aplicada y del Doctorado en Investigación de la Comunicación (PNPC) de la Facultad de Comunicación de la Universidad Anáhuac México. Doctora en Ciencias Sociales por la Universidad Iberoamericana. Maestra en Educación por la Universidad de Stanford y licenciada en Comunicación por el Instituto Tecnológico y de Estudios Superiores de Occidente. Coordinadora de textos como Comunicación Estratégica en las organizaciones; Ética, medios y democracia; entre otros. 
ya que el primero aprende a analizar hacia dónde quiere llegar y lo que pretende demostrar, además de que motiva al alumno a tener un juicio crítico. Por su parte, el alumno desarrolla un liderazgo participativo, un pensamiento crítico, una actitud de cooperación, de intercambiar ideas y de adquirir nuevos conocimientos.

\section{Palabras clave}

Estudios, casos, metodología, aplicación, beneficios.

\section{Introducción}

Desde el siglo pasado, la metodología del caso es una técnica muy utilizada en el proceso educativo, debido a la gran oportunidad que ofrece a aquellos que participan en su análisis y reflexión, además de los beneficios para el desarrollo del pensamiento crítico, de trabajar en equipo y de ejercitar el manejo de toma de decisiones, por lo que se puede afirmar que esta metodología ofrece un aprendizaje real y significativo.

Esta investigación pretende dar respuesta a las siguientes interrogantes: ¿En qué consiste el método de casos? ¿Cómo se origina? ¿Qué utilidad genera esta metodología? ¿Para qué sirve el método del caso? ¿Qué elementos se necesitan para elaborar un caso? ¿Cuáles son las dificultades y barreras que se presentan en la metodología de casos?

\section{¿En qué consiste el método del caso?}

Christensen Roland y Abbey Hansen (1984, citado en Gómez y Villazón, 2003) en su obra Teacbing and the case method comentan que un caso típico es el registro de un problema administrativo que fue encarado por ejecutivos de negocios, junto con las circunstancias que lo rodearon, y las opiniones y prejuicios de los que dependió la solución que dieron los ejecutivos. Este caso real y particularizado es presentado a los alumnos para que lo analicen con cuidado, lo discutan abiertamente y decidan qué tipo de acción se debió tomar.

Por su parte, García Galindo (1995) define el método del caso como un diálogo sistemático en donde se analizan situaciones reales, con objetivos y propuestas que se presentan del caso, y en donde participan los estudiantes de manera activa y cooperativa. Por lo antes mencionado, se puede afirmar que en la metodología de casos existe una interacción entre 
alumnos y maestro, un diálogo que se centra en situaciones reales con fines de aprendizaje, pero que debe ser sistemático, con una metodología para tratar de dar solución a una situación o problema que se presenta en la realidad. De esa manera, el objetivo de la metodología de casos es que los alumnos generen soluciones específicas para un problema que se presenta en la vida real, que puede ser de una persona, familia, grupo o empresa.

\section{¿Cómo se origina la metodología de casos?}

El estudio del caso como estrategia didáctica tiene su origen a finales del siglo XIX en la Escuela de Leyes de la Universidad de Harvard. En un principio, el objetivo de implementarlo en dicha escuela era que los estudiantes de derecho buscaran una solución a un problema de la vida real, con una historia concreta, y tomaran el papel de defensores u opositores (Gómez \& Villazón, 2003).Pasaron pocos años y en 1935 se implementa como método docente en las diversas escuelas y facultades de Harvard.

Al transcurrir los años, el uso del estudio de caso se extiende a diversas partes del mundo, en Europa, Asia y América, así como a diferentes campos, como la administración, la medicina, las ciencias políticas, la comunicación y la ingeniería, entre otros.

El método del caso en México tiene ya varias décadas de haberse implementado. Se usa como una metodología didáctica en diferentes universidades y centros de investigación, entre los que se puede citar a la Universidad Anáhuac México, al Centro de Investigación en Comunicación Aplicada, al Instituto de Capacitación de Alta Dirección, a la Universidad Panamericana, al Instituto Autónomo de México, al Centro de Investigación México-Estados Unidos, así como a la Universidad Nacional Autónoma de México.

Cabe señalar que la industria cinematográfica lo ha representado en diversas películas, como El cliente, dirigida por Joel Schumacher y con la actuación de Susan Sarandon y Tommy Lee Jones; La fuerza de la verdad, dirigida por Mike Nichols, con Harrison Ford y Annette Bening; y Legalmente rubia, dirigida por Robert Luketic y estelarizada por Reese Witherspoon y Matthew Davis.

\section{¿Qué utilidad genera esta metodología?}

El objetivo principal de quienes utilizamos la metodología de casos es preparar alumnos, capacitarlos para la toma de decisiones bajo las presiones y problemas que se presentan en el acontecer diario. No pretendemos formar bibliotecas andantes, sino preparar hombres de 
acción que serán medidos y calificados por objetivos, estrategias y metas cumplidas. Por lo tanto, éstos son los beneficios que obtiene el catedrático al aplicar la metodología del caso:

- Al definir los objetivos que se quieren alcanzar con esta metodología, el catedrático aprende a analizar hacia dónde quiere llegar y lo que pretende demostrar.

- Con este método el catedrático descubre y desarrolla su propio sistema, con la finalidad de entender la posible solución al problema.

- El catedrático motiva al alumno a formarse un juicio crítico y presentar posibles soluciones a la problemática planteada.

- Se motiva a los alumnos con un aprendizaje significativo, basado en hechos reales.

A su vez, el alumno obtiene estos beneficios:

- El estudiante podrá obtener un liderazgo participativo, en donde escucha a los demás, pero también es partícipe en la toma de decisiones y posibles soluciones.

- Con la metodología de casos el alumno desarrollará un pensamiento crítico, de análisis y síntesis.

- Se formará para trabajar en equipo, así como para desarrollar una actitud de cooperación, intercambio y respeto a las ideas de los demás.

- Con esta metodología el estudiante no memoriza, sino que se le invita a tener un acercamiento a la realidad, a analizar las necesidades del contexto y a enfrentar los problemas en el mundo real.

- El estudiante analizará la realidad y podrá adquirir nuevos conocimientos.

\section{¿Qué elementos se necesitan para elaborar un caso?}

Antes que nada, es necesario seleccionarlo, para lo cual intervienen elementos personales, tales como los temas de interés, el tema de la clase, la utilidad, la identificación del caso que servirá para cumplir algún objetivo específico de la materia impartida, entre otros; por ello es conveniente plantearse la siguiente pregunta: ¿Cuál es el papel que desempeñará este caso dentro del curso?

Después es necesario conocer el contexto en el que se desarrolla el caso y recordar que si no se tiene un conocimiento de la realidad, de lo que rodea al problema, del entorno social, político y económico, el alumno y el educador no podrán cumplir los objetivos. Una vez que se selecciona el contexto se pasa a la investigación, a la recopilación de datos a través de 
fuentes primarias, documentos, bases de datos que tengan una relación directa e indirecta con el caso.

Finalmente, se procede a la investigación de campo con herramientas cualitativas que van desde entrevistas, observación, focus groups, hasta herramientas cuantitativas como cuestionarios y encuestas. En este punto se requiere una mente inquisitiva, buscar las diversas opiniones, hablar con las partes involucradas; hay que tomar en cuenta que esta investigación tiene por objetivo conocer y comprender el caso en sus diferentes facetas, considerando los diversos actores que intervienen en el mismo.

\section{¿Cómo se escribe?}

Como se comentó anteriormente, primero es necesario seleccionar el caso, después hacer la investigación y recolectar la información, y asíllegamos a la parte de redactarlo. Para ello se sugiere un esquema de los temas que se van a desarrollar. Al igual que en un libro, se presentan capítulos y subcapítulos, al escribir un caso se diseña una estructura, que es una forma de organizar los datos y de seleccionar la información que se considera más relevante. Es necesario tomar en cuenta que la clasificación de los temas puede ser por orden de importancia y utilizando el método deductivo, que parte de lo general a lo particular.

Gómez y Villazón (2003) recomiendan la siguiente estructura para elaborar el índice de casos: introducción; marco contextual; antecedentes y datos generales de la empresa que se va a estudiar; cuerpo del caso; propuestas, estrategias y soluciones; notas didácticas; anexos. A continuación, se presenta una breve descripción de cada parte de esta estructura.

Introducción. Su nombre lo dice todo: introduce al lector en lo que se va a presentar, cuál es la empresa a estudiar, cuál es el problema a tratar. Es importante destacar que la persuasión juega un papel importante en este punto, pues es el anzuelo que capta la atención del lector. En la Harvard Business School muy frecuentemente se utiliza en la introducción de los casos el diálogo y la descripción del personaje como recurso, por lo que se recomienda como técnica la observación y la entrevista directa o indirecta con alguna persona involucrada en el caso.

Marco contextual. Quien realiza el caso, ya sea el profesor o el alumno, presentará el entorno que lo rodea; esto se refiere al contexto, a lo que se observa alrededor del mismo, la situación económica, política, social. 
Antecedentesy datos generales de la empresa a estudiar. Cuando se estudia un caso es conveniente referirse a los antecedentes y datos generales de la empresa, explicar su origen, cuál ha sido su desarrollo, la misión, visión, los valores, la filosofía, así como el organigrama.

Cuerpo del caso. En este punto, para presentar el cuerpo del caso, se agrupa la información en orden lógico, se presenta el problema de lo general a lo particular y se va enlazando el organigrama del punto anterior; hay que identificar los departamentos, sus funciones y señalar en dónde se presenta el problema y cómo afectó a los diversos departamentos o direcciones.

Propuestas, estrategias y soluciones. Una vez analizado el problema o los problemas, conviene presentar las estrategias, los planes de acción que se llevarán a cabo y cuáles son las propuestas con respecto a la solución del problema.

Nota didáctica. El caso debe incluir una guía del profesor o nota didáctica dirigida al maestro que quiera utilizar el caso. Por lo regular esta guía contiene los siguientes puntos:

- Objetivos pedagógicos del caso

- Una lista de los temas que cubre el caso

- Una serie de preguntas que se proponen para discusión

- Respuestas posibles a estas preguntas

- Información adicional que será de utilidad para el profesor.

Anexos. Información que es de utilidad para conocer el caso; dentro de ello se encuentran datos numéricos, listados, relación de catálogos.

\section{¿Por dónde empezar?}

Al observar la estructura, así como la redacción de los casos internacionales y nacionales que se publican en la Universidad Anáhuac México y en la Harvard Business School, se recomienda que los casos se escriban en tiempo pasado, puesto que son problemas que ya se presentaron y que, al establecer estrategias y propuestas, ya fueron resueltos en su momento. Asimismo, se recomienda tener presentes los siguientes puntos:

- Crear una trama interesante con una buena estructura

- No incluir en el texto opiniones del autor 
- Expresar con claridad los títulos y subtítulos

- Sólo podrán utilizarse modismos en situación de diálogo o en las entrevistas que se presenten de manera textual.

\section{¿Cómo llevarlo a cabo en el salón de clases?}

En cuanto al proceso operativo, Martínez y Musitu (1995) cuentan con el decálogo que a continuación se presenta:

1. Estudiar el caso: en el contexto en el que se desarrolla.

2. Analizar el caso desde diversas perspectivas, identificar las variables que describen al mismo.

3. Identificar la información primaria y secundaria y analizar los datos principales y adicionales del caso.

4. Detectar los puntos fuertes y débiles de la situación:las interacciones y planteamientos teóricos.

5. Estudiar cada uno de los problemas, describir los cambios que se tienen que llevar a cabo en cada una de las situaciones para solucionar los problemas.

6. Generar diversos planes u opciones de acción para llevar a cabo cada uno de los cambios.

7. Estudiar a fondo los pros y los contras de cada alternativa, seleccionar las que sean factibles y establecer un proceso de selección riguroso, hasta elegir la que tenga el menor número de dificultades y efectos negativos.

8. Implementar la decisión elegida y plantear las estrategias y recursos que se necesitan para llevarla a buen término.

9. Determinar el cómo o los planes de acción que se llevarán a cabo y la manera en que se evaluarán las decisiones elegidas.

10. Reflexionar sobre los temas teóricos que plantea el caso presentado.

Pero no todo es miel sobre bojuelas; algunas personas del campo de la enseñanza consideran que la metodología de casos es una técnica complicada, propia de expertos. No obstante, se trata de un sistema de enseñanza efectivo, pero poco conocido y aplicado. Si bien es cierto que esta metodología requiere una preparación específica del catedrático, pues no se trata de un método de instrucción sino de formación en profundidad -que genera en los profesores y estudiantes capacidad de análisis, de reflexión, de toma de decisiones-, son más sus 
ventajas que las dificultades. A continuación se presentan algunos elementos que pueden representar esas dificultades:

- Cuando los grupos son numerosos, se puede llegar a perder el control de los mismos.

- Algunas veces los alumnos se apasionan con el caso y no aceptan respuestas de sus compañeros, por lo que el catedrático debe llamar al orden.

- Los alumnos, sobre todo los más jóvenes, pueden tener la percepción de que hay poca o nula relación del caso con los contenidos de aprendizaje del curso.

- Como no se trata de una metodología tradicional, es difícil que algunos padres de familia la acepten como estrategia didáctica.

- El profesor debe tomar en cuenta que el caso debe versar sobre un tema que involucre a todo del grupo; de lo contrario, algunos alumnos pueden perder el interés de participar en las discusiones.

- Los casos con finales abiertos, con diferentes propuestas de solución, pueden ser difíciles de aceptar para algunos alumnos, ya que no se observan respuestas hechas, respuestas correctas.

- El profesor debe tomar en cuenta el tiempo de discusión del caso para administrarlo adecuadamente; de lo contrario se puede perder el interés del grupo.

En suma, es importante considerar el método del caso como una herramienta para el proceso enseñanza-aprendizaje que puede ser muy útil para el desarrollo de competencias en los alumnos de distintos grados y disciplinas. Su éxito dependerá de la definición clara de su objetivo, de su correcta planeación y de la ejecución de la dinámica de solución y desempeño de los alumnos.

\section{Referencias}

García, M. (1995). Administración de las relaciones laborales. México: Siglo XXI.

Gómez, D. \& Villazón, G. (2003). El método del caso: una herramienta útil para el estudio de las empresas. Thomson Business Fournal, I, 129-133.

Martínez, A. \& Musitu, G. (2004). El método del caso. Guía rápida para las nuevas metodologías. Servicio de Innovación Educativa de la Universidad Politécnica de Madrid, España. Recuperado el 15 de julio de 2016 de https://cb.hbsp.harvard.edu/cbmp/pages/home

Roland, C. \& Hansen, A. (1984). Teaching and the case method. Massachussets: Harvard Business School Publishing Division. 